\title{
Injury to Liver
}

National Cancer Institute

\section{Source}

National Cancer Institute. Injury to Liver. NCI Thesaurus. Code C35241.

Damage to liver structure or function due to trauma or toxicity. 